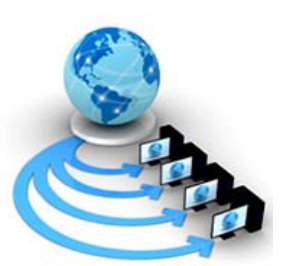

International Journal of Advanced Research in Computer Science

RESEARCH PAPER

\author{
Available Online at www.ijarcs.info
}

\title{
THE CURRENT STATUS AND RESEARCH IN INDUSTRIAL BIG DATA ANAL YSIS IN SMART INTELLIGENT SYSTEMS
}

\author{
G. Sunil \\ Assistant Professor, \\ Department of Computer Science \& Engineering, \\ S R Engineering College, \\ Ananthasagar, Warangal, \\ Telangana, India,
}

\author{
Sallauddin Mohmmad \\ Assistant Professor, \\ Department of Computer Science \& Engineering, \\ S R Engineering College, \\ Ananthasagar, Warangal, \\ Telangana, India
}

Kanegonda Ravi Chythanya

Assistant Professor,

Department of Computer Science \& Engineering,

Sree Chaitanya College of Engineering,

Karimnagar, Telangana, India,

\begin{abstract}
Intelligent manufacturing has become a point of reference in Cyber-Physical Systems (CPS) and produced a revolutionary change in that field. That created network will produce the large amount of data. The intelligent manufacturing has the characteristics as highly correlated, deep integration, dynamic integration and huge volume of data. As a consequence it still faces numerous challenges. We recapitulate and analyze the research scenarios in both domestic and aboard in the current environment, including industrial big data collection, the predictive diagnosis based on industrial big data, group learning of product line equipment and the product line reconfiguration of intelligent manufacturing. In this Paper, we propose the research strategies, including acquirement schemes of industrial big data under the environment of intelligent and deduction method based intelligent product lines, predictive diagnostic methods on production lines based on the research status and the problems based deep learning among devices based on cloud supplements and 3D self-organized reconfiguration mechanism based on the supplements of cloud.
\end{abstract}

Keywords: Intelligent data, Cyber-Physical Systems, Big Data, Cloud Computing, 3D self-organized mechanism.

\section{INTRODUCTION}

In the early ages the ability to initiate the strategy of United States in "Industrial Internet" as well as the strategy in Germany "Industry 4.0" the book "Manufacturing White Book of the Year 2014" which was published in japan and the plan "Made in china 2025" formulated and issued in china have made an intelligent manufactured an orientation which was supported with the high priority by their nations.

That is very important to establish the intellectual factories based up on the industrial big data and the "Internet Plus"[1]. Presently the producing field still faces numerous international Challenges for suppose with the support of information technologies coming up (e.g., cloud computing and wireless networks) implementation of metaphysics modelling towards the intelligent producing product lines the activity designation, optimisation and reconfiguration on intelligent product lines through industrial big data have essential research values[2][3].

The investigation and application for the upcoming generations of manufacturing technologies to boost the pliability of intelligent manufacturing product lines and also the utilization potency of the resources have vital theoretical significance[5].

An intelligent manufacturing plant regards the CyberPhysical Production System (CPPS) as its kernel and it takes a deep fusion of manufacturing technology as its characteristics that will combine the future generation of the information technologies such as Big data, IOT and cloud computing into various stages of producing process so as to understand the highly adequate production of intelligent products with customization as its characteristics[4]. From information technology the manufacturing domain has various features the requirements such as real-time, reliability assurance during the process of manufacturing the product[5]. It will not satisfy the demands of intelligent factory under the new frame of manufacturing by applying the sophisticated techniques. The current manufacturing equipment does not have configurability and is designed to deal with the similar type of products during the manufacturing process the equipment does not support the active operation and also maintenance likewise dynamic reconfiguration[4]. Therefore the customised products proposed high-efficient manufacturing of new requirements on the configurability of manufacturing equipment's and production line such as fault analysis of devices and manufacturing systems development and reconfiguration[6][7]. It needs the basic technologies on the foundations of the current information technology in order to adapt the requirements of intelligent factories.

Compare to the traditional approach of production the intelligent manufacturing has the following common characteristics: 
- Highly Correlated:

The knowledgeable manufacturing of systems endure under a network surroundings in which construct/detect and assemble devices transmission system work piece server and surveillance terminals are all interconnected through various types of networks such as wireless cable and real-time/non real time to Convey and exchange the data with each other.

\section{- Large amount of data:}

The manufacturing of system should satisfy the tiny batch of personalization. In order to produce huge amount of data counting the health position of the equipment the status of manufacturing process and the commodity report each type of intelligent substance requires real-time negotiation on rehabilitation[4]. The operations of high speed network technology big data processing technology enable transmission cloud computing technology in search of large amount of data repository[3].

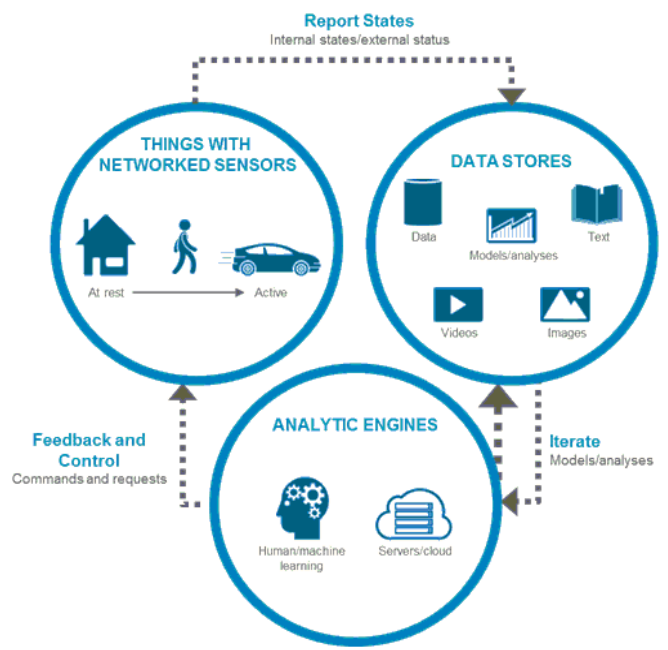

- Wide Integration:

The intelligent physical substance in the bottom layer and the super vision terminal in the uppermost layer are interconnected with the cloud platform and interconnected with various types of data in the system uploaded form the industrial Big Data[2].Thereby it can simultaneously achieve control physical operations in a network and data processing crack the information boundary of each procedure and recognize the wide integration of information environment and physical environment which is cyber-physical system[3].

\section{- Dynamic Reconfiguration:}

It is important to determine the transmission paths and require type of devices according to the health condition of equipment and the various types of work piece in order to accept the adequate production of different type and small batch products for the healthy position of the equipment and the dynamically varying type of work piece, it is necessary to rebuild the system sources during the functions of systems.

The foundation of big data processing with the cloud platforms and high degree interconnection based on the network. The mechanism of dynamic reconfiguration which are related to intelligent product lines are the important points of flexibility and highly efficient manufacturing where as the new methods on the industrial big data analysis bring conditions for diagnosis and the optimization of integration of intelligent manufacturing product lines[3][7]. Presently, the research on the CPS and intelligent manufacturing are in ascendant. However the recognition of intelligent manufacturing in the current situation is still facing many problems related to present situation of both domestic and abroad[8]. Some of the crucial unsolved scientific difficulties are utilizing the analytical technique on industrial big data to update the performance of interconnected environment personalize the need of the manufacturing system[6].

It includes the following aspects they are:

- The present research status of individual big data analysis of smart factory in both domestic and broad are analysed and summarized[8].

- The research approach for industrial data analysis which involves the acquisition design, ontology modelling, predictive diagnostic methods that are based on the nature network .

\section{THE PRESENT RESEARCH STATUS IN BOTH DOMESTIC AND ABROAD:}

The study of project based on both out home and abroad, which is of five aspects Big data collection designing of the intelligent product lines based on philosophy the industrial big data of predictive diagnosis Associative learning product line equipment and the product line reconfiguration of intelligent manufacturing[5][8].

\section{A. INDUSTRIAL BIG DATA COLLECTION:}

The speedy growth of micro electronics technology embedded technology control and measurement technology and distribution technology has achieved am important growth at home and abroad in data collection methods and data processing techniques[2]. The background application of Internet of Things (IoT) and of wireless sensor network (WSN) and communication have been a most interesting topic of investigations in the previous years and the most commonly used technique for the acquisition of data processing is data fusing[9]. The one who proposed the Kalman algorithm and applied it in the industrial IoT to operate data fusion processing is Chun-mei wang. The selfadaptive data fusion algorithm is proposed by the Prie to enlarge the positioning accuracy. The data of equipment products, conditions the manufacturing the environment of intelligent manufacturing product lines. These data have the real-time alarms and equipment logs and periodical signals etc. The product lines which are associated with big data are shared in various locations of manufacturing environment and there are various types of data designs of the traditional data aiming at the same signal of various data sources do not arrange better solutions to the problem of capable acquisition of big data from the producing product lines[3][10].

In the recent years the Industrial IoT and the concept of OCPU have provided advance approach for the big data acquisition and information interactions of product lines based on the manufacturing environment is defined by the software the data acquisition nodes that are capable to taking all type of data inputs by the means of dynamic configuration of software that has strong flexibility[11]. 


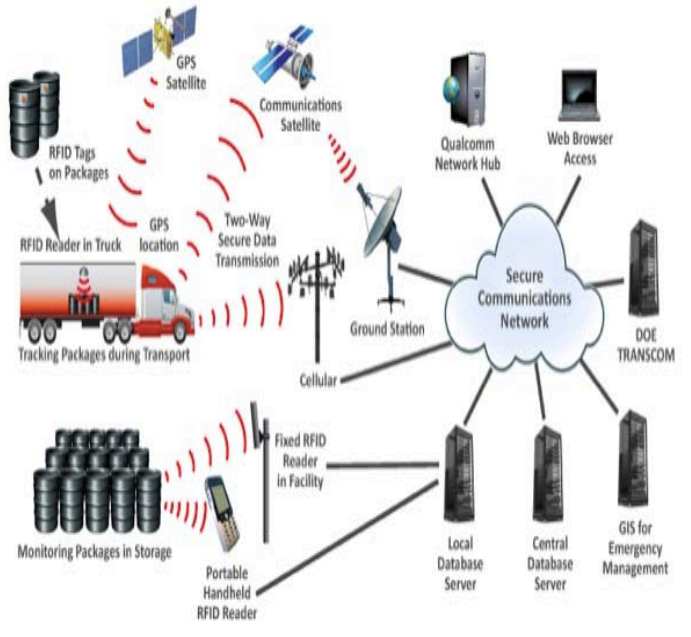

\section{B. RELATION ORIENTED MODELLING TO MANUFACTURING ENVIRONMENT:}

The relation oriented modelling implements modelling method and deduction mechanism towards the concepts and the mutual relations of precise domain[11]. Which are applied in article intelligence semantic network and software engineering that are widely applied in the domain of information science. The brand new visual angles to the realization of detection alarm and performance optimization where resource allocation of product lines there are the specific applications which are in the modelling of ontology. The multiple influence factor synthetic weighting and the semantic increment achieving precise retrieval results are introduced[12][13]. Big Data aimed at the domain of IoT and implemented analysis by dividing relation into five major classes[4]. Within comprehensive philosophy oriented to knowledge representation that define the view of relation, service, measurements and observation. In order to provide relatively better solutions to the problem of IoT expansion due to heterogeneous data, applied statistical learning and the method of ontology to design a new middleware architecture of IoT[13]. The mentioned designs attain the modelling establishment and initial reasoning but they are unable to provide adequate solutions to the key problems such as performance optimization and resource allocation under the circumstances of manufacturing these designs provide brand new styles of thinking on the diagnosis and reconfiguration of product lines[14].

\section{DIAGNOSIS BASED ON BIG DATA}

The contemporary scale of industrial system is growing and the corresponding increase defects are determining the reliability and the effectiveness of the system which makes the fault diagnosis and disclosure on all the sensors for the screw chillier[13]. System based on the support vector data depiction algorithm in order to solve the outcome conflict and classification of efficient problems of support vector machine (SVM) with the fat development of datastores, networks and data acquisition capacities the traditional schemes on fault prediction and designation in the model training, are unable to endorse to the present large volumes and high-rate of data[14][15]. In the recent years, domestic and foreign scholars have detected that learning her peculiar effects in big data processing of numerous fields update the accuracy rate of perception of images net images from $74 \%$ of normal intelligent method to $83 \%$ by building the deep learning model in order to utilize the deep learning model to inquiry exotic particles in high energy physical and determine that the deep learning could enlarge the searching capabilities of colliders[14] . So that some professor applied the deep learning model into predictive diagnosis of error in order to fascinate the requirement of predictive diagnosis in even large manner under in history of big data[2]. The one who proposed the multi-sensor health diagnosis model based on deep learning model and used the case scrutiny to determine that deep belief network categorization model. In the diagnosis of complex system displace better diagnosis perform in divergences with other categorization models was innovatively proposed Based on the energy operate and deep believe network the faults diagnosis method on the reciprocating the proposed model is highly predictive and versatile to industry reciprocating appliances to correlation vector machine and BP neural network have proved the analysis failure classification perform[15].Aiming at present status that fault diagnosis of current sensing electric motors adopts supervise learning to extract the features of faults a deep network that method the noise removal coding into inadequate automatic code realize the features radiation of unsupervised learning applied that to the fault diagnosis sensing electric motors[16][17]. The above mention predictive diagnosis method based on deep learning common assume that the training date is tagged data and probably are unable to gravity the necessity of producing big data in product line[7]. One original signals a predictive diagnosis model can perform training which will have specific importance.

\section{GROUP LEARNING OF THE EQUIPMENT ON PRODUCT LINE:}

The surrounding of intelligent manufacturing they introduced the earlier configuration experience of related working procedure obtain the group learning of working procedures that will signification the working ability of equipment on product line and shorten intensity of maintenance from both the domestic and abroad organize several investigation towards the self-adaptive learning group learning and method relative to the devices in recent year from scholars such as mobiles robots industrial robotic arms and have gained some achievement . The mechanical arms utilized the radical bases function (RBF)with completely unknown parameters and designed a new selfadaptive neural control algorithm in the joint space. They integrated typical application of different class robot task and keep the codes of running task that familiarized to multi class robots, industrial fields and academy attention are considerably attached[9][13].In order to exploit Q-learning and cascaded support vector machine (SVM) to facilitate the robot to have the capability of learning was proposed by S.W.SEO. In addition to these it focus to grab mechanical hand multiple research institutes make their own database public, like the KIT object data set in Massachusetts institutes of technology[14]. All of the above described studies, by the association with cloud computation and big data pursue to realize the technical distribution towards the robot in the bottom layer in cloud terminal[15]. Under 
environment of producing, they are also huge amounts of product line devices which don't integrate hardware like embedded equipment doesn't have the learning ability of code-level technical skills. The similar types of equipment to build a practical example of cloud terminal, co-related and calculate the behavior of similar product through the snap shooting technology[3][4]. Optimized the configuration of based layer in order to develop the flexibility and selfadaptability of the equipment. Based on the curve fitting and clustering the prediction mechanism of remaining effectively span based on the prior samples presently the main intention of multiple scientific search team both in aboard and domestic are still to in make the trap of learning an all type of robot equipment as a dominate tasks and to authorize the learning mechanism all sorts of product line equipment under the manufacturing of environment that will have symbolic imported in attaining intelligence and flexible manufacture \& maintenance[16].

\section{E. RECONFIGURATION OF INTELLIGENT MANUFACTURING PRODUCT LINES}

The dynamic reconfiguration of manufacturing systems commonly seen as an imported attribution on the system for on the system for handling with inter/outer big date factors and enhancing the elasticity of itself[17]. The dynamic reconfiguration of manufacturing system fascinates considerable attention from the academies both at aboard and home in recent years. The one who contracted the selforganized unity by absolutely utilizing the approach like SDN OPC uniform structuring[19]. The frame work earned the reconfiguration of producing through the function of adding and removing develop proposed a efficient selforganization based on learning automated machine theory the response threshold design to deal with tasks allocation in multi robots system[15].The needed amount, layout and work load of equipment when external demand various by establishing different mathematical models in order to develop smooth reconfiguration process of unity manufacturing system[14][17]. In the dynamic manufacturing unit when equipment is not predictable the one who established multi stage scheme model and supplemented it with several universal inspiration deduction models to solves the problem unit manufacture system of in accurate equipment in severe prime cost all above illustrated research studies have some type of refer valves towards the study of research project the difficult problem which are unsolved till existed on the reconfiguration mechanism of intelligences production line with know and unknown failures[10].

\section{ANALYSIS STRATEGIES:}

Intelligent manufacturing has its own features and additionally brings some issues. The analysis may be divided into 5 aspects: Acquisition designs of business massive information beneath the setting of intelligent manufacturing, ontology modelling and deduction methodology[19]. Primarily based on intelligent product lines predictive diagnostic strategies on production lines based on deep learning among all the devices based on the deep neural network deep learning among the devices is possible on cloud supplements and supplements of cloud where the three dimensional self-organized reconfiguration mechanism is based[2][17].

Here, The figure 1 below shows the key technologies for Big data analysis in smart factory.

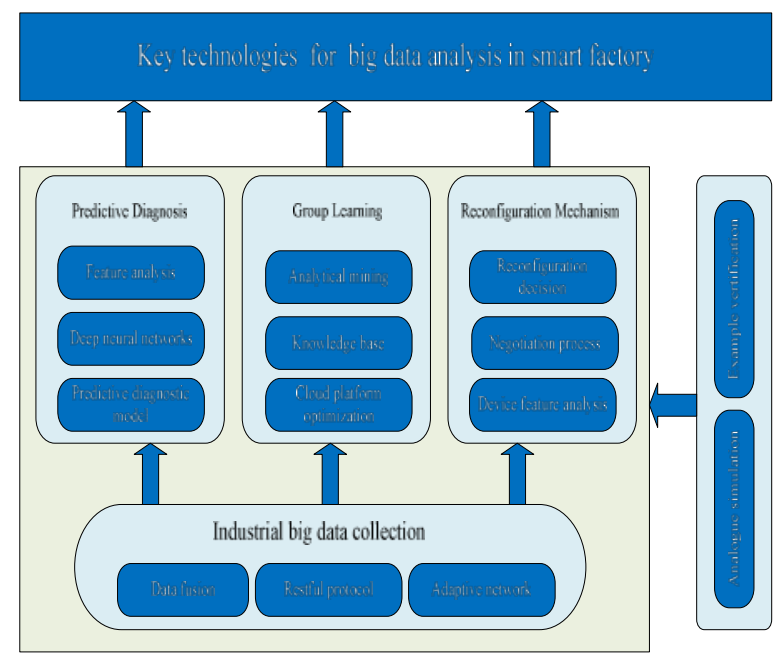

Figure 1. Key technologies for big data analysis in smart factory

1. Acquisition Designs of Industrial Big Data under the Environment of Intelligent Manufacturing

In an environment of intelligent manufacturing the standard sensing of information fusion models does not seem to be capable of achieving economical acquisition of huge information within the manufacturing product lines[3]. The proposed concepts of software-defined sensing networks and software defined IoT in recent years have provided new directions of thinking in massive information acquisition on the assembly lines within the manufacturing setting[7][12]. They adopt the software package defined information acquisition nodes by the means of dynamic configuration from the software which adapt data inputs of different characteristics and different types and displays strong flexibility[19]. The authors decided to study and work on the issues as follows: industrial massive information fusion models under the setting of intelligent manufacturing information acquisition nodes supporting[16].The Restful web page service protocol self-adaptive network bandwidth allocation algorithm based on software definition; software defined data acquisition method and data interaction among the supported OPC UA[14].

2. Ontology Modelling And Deduction Method Based Intelligent Manufacturing Product Lines:

3. The intelligent manufacturing emphasis the high flexibility of manufacturing process the scheduling of product lie resources and the optimization of manufacturing performance[13]. The complicated categories of equipment in the product lines and the continuous expansions of equipment due to these it is unable to handle the intelligent manufacturing systems[17]. So as to realize the versatile resource allocation, detection alarm and performance improvement of intelligent manufacturing line of products[12][18]. On the related problems the authors perform analysis: the association of intelligent base and database end data with semantics: an Ontology and 
restrictions where inference mechanism is based on realization principles of warning detection, resource allocation and performance optimization based on ontology and inference[18].

4. Predictive Diagnostic Methods on Production Lines based on Deep Neural Networks

5. Now a day's modern devices have sophisticated structures and there reciprocally coupled extremely nonlinear dynamic characteristics among multiple devices[9]. Moreover the fault issues square measure totally different completely different under different scenes and therefore the characteristics of signals to be processed also different[12][16]. So as to confirm high potency background of commercial massive information a prophetic diagnostic technique that's universally elastic to multiple information is projected[17]. The authors committed to conduct analysis on the subsequent aspects: feasibleness analysis of deep learning models applicable to the prophetic deep neural networks analysing effective and universal compatibilities of strategies on predictive diagnostic methods[19].

6. Group Learning among Devices based on Cloud Supplements

The group learning among the devices based on cloud supplements is shown in the figure below. The complex oriented tasks are different due to the different degrees of hard ware integration on product line leveled devices[3]. Concerning to the devices with high degrees of hardware integration the potency on task execution of devices will be considerably upgraded by learning the expertise of comparable task[2]. Whereas concerning the devices with low degrees of hardware integration parameter configuration and therefore the resultant work procedures of devices will be optimized by learning the historical standing data of comparable devices[2][3]. The authors commit to conduct comprehensive study on the subsequent problems improvement of personal cloud platform supported the setting of intelligent establishing the dynamic knowledge domain headed to specific task at the cloud terminal for devices facing high degree of hardware integration[2][14]. The knowledge domain data are updated when learning and execution the 'coding level' knowledge by the devices establishing the log of standing data on the devices [1][16].

The three-dimensional self-organized reconfiguration mechanism based on the supplement cloud[1][2][4]. Under the intelligent manufacturing the known and unknown errors of intelligent devices will trigger the events of product line reconfiguration. Whereas activity reconfiguration, adopting the reconfiguration strategy supported organization and cloud supplements of intelligent devices can make sure the action of expected effects on the performance of product lines[5]. The authors committed to conduct a descriptive analysis on the subsequent subjects: on intelligent devices task particular devices; the coordinating method of device-oriented behaviours, the logic series of efforts and reconfiguration on physical locations of devices, the transaction design on interaction of intelligent devices and cloud levels and therefore the inactivation management models of product lines[3][19].

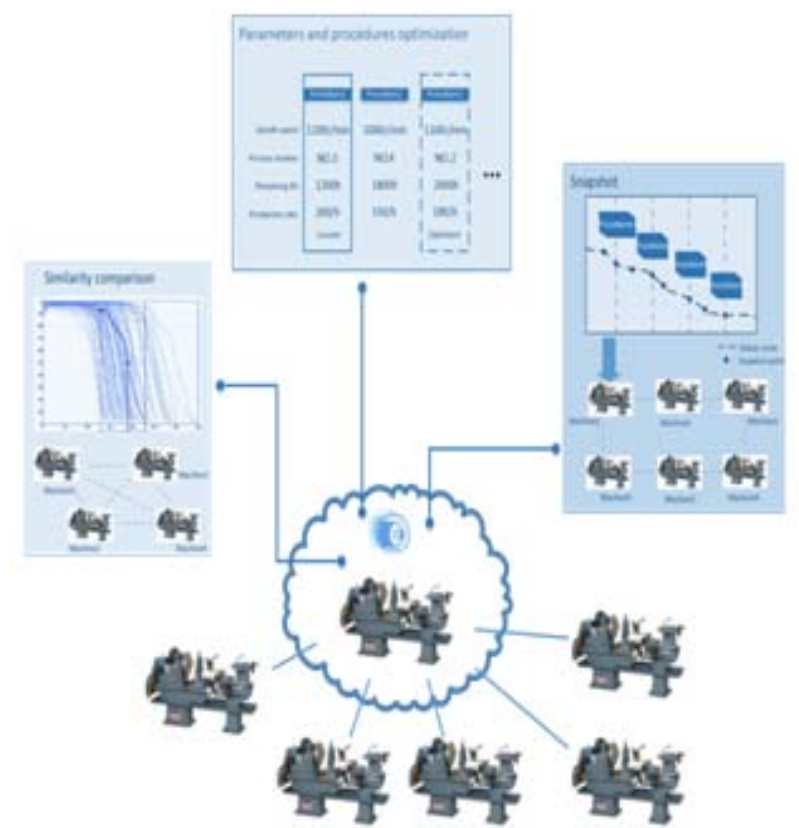

Figure 2. Group learning among devices based on cloud supplements

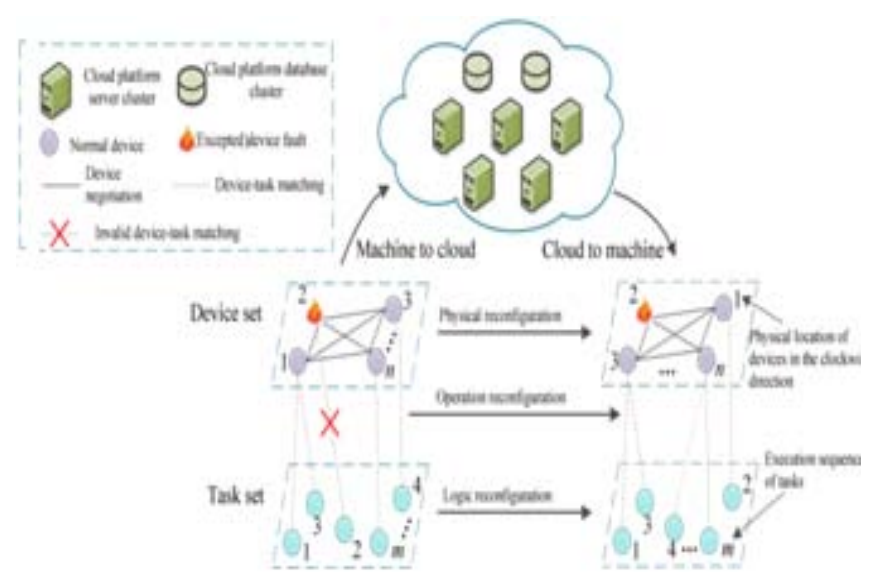

Figure 3. Three-dimensional self-organized reconfiguration mechanism based on the supplements of cloud

\section{CONCLUSION:}

In this page we have a tendency to first comprehensively review and study the standard reception and abroad from the aspects of business large knowledge assortment, modelling of the intelligent product lines supported ontology the predictive diagnosis based on the industrial big data combined learning of product line equipment and the product line reconfiguration of intelligent manufacturing in this way we reveal the problems and challenges which are faced. We also point the insufficiency of data analysis under the traditional manufacturing environments and propose our research plans and the detailed format of some aspects as follows: acquisition models of industrial big data under the environment of intelligent manufacturing intelligent product lines on deduction methods production lines based on deep neural network on predictive diagnostic cloud supplements on deep learning among devices and supplements of clouds in the three-dimensional self-organized reconfiguration mechanism. It helps in suggesting thoughts for further studies. 


\section{REFERENCES}

[1] J. Wan, C. Zou, S. Ullah, C. Lai, M. Zhou and X. Wang "Cloud enabled Wireless Body Area Networks for Pervasive Healthcare,” IEEE Network, vol. 27, no. 5, pp. 56-61, 2013.

[2] W. Yuan, P. Deng, T. Taleb, J. Wan and C. Bi, “An Unlicensed Taxi Identification Model based on Big Data Analysis,” IEEE Transactions on Intelligent Transportation Systems, vol. 17, no. 6, pp. 1703-1713, 2016.

[3] J. Wan, S. Tang, D. Li, S. Wang, C. Liu, H. Abbas and A. Vasilakos, "A Manufacturing Big Data Solution for Active Preventive Maintenance," IEEE Transactions on Industrial Informatics, vol. 13, no. 4, pp. 2039-2047, 2017.

[4] D. Zhang, Z. He, Y. Qian, J. Wan, D. Li and S. Zhao, "Revisiting Unknown RFID Tag Identification in Largescale Internet of Things,” IEEE Wireless Communications, vol. 23, no. 5, pp. 24-29, 2016.

[5] Z. Shu, J. Wan, D. Zhang and D. Li, "Cloud-integrated CyberPhysical Systems for Complex Industrial Applications," Mobile Networks and Applications, vol. 21, no. 5, pp. 865-878, 2016.

[6] D. Zhang, Y. Qian, J. Wan and S. Zhao, "An Efficient RFID Search Protocol Based On Clouds," Mobile Networks and Applications, vol. 20, no. 3, pp. 356-362, 2015.

[7] J. Wan, D. Zhang, Y. Sun, K. Lin, C. Zou and H. Cai, "VCMIA: A Novel Architecture for Integrating Vehicular Cyber-Physical Systems and Mobile Cloud Computing," Mobile Networks and Applications, vol. 19, no. 2, pp. 153160, 2014.

[8] L. Wang, Z. Shang, and W. Wang, "The development and application of the data acquisition system," Electrical Measurement \& Instrumentation, vol. 41, no. 8, pp. 4-8, 2004.

[9] J. Dong, D.F. Zhuang, Y.H. Huang, et al, "Advances in multi-sensor data fusion: algorithms and applications," Sensors, vol. 9, no. 10, pp. 7771-7784, 2009.
[10] M. Wang, “ Data fusion processing of the Internet of industrial things based on adaptive unscented Kalman algorithm,” Electric Drive Automation, vol. 38, no. 4, pp. 43-47, 2016

[11] P. Huang, T. Chen, C. Wang, et al, "Compressed sensing algorithm based on data fusion three in wireless sensor networks," Journal of Electronics \& Information Technology, vol. 36, no. 10, pp. 23642369, 2014.

[12] J. Prieto, S. Mazuelas, A. Bahillo, et al, "Adaptive data fusion for wireless localization in harsh environments," IEEE Transactions on Signal Processing, vol. 60, no. 4, pp. 1585-1596, 2012.

[13] J. Wan, S. Tang, Z. Shu, et al, "Software-defined industrial internet of things in the context of industry 4.0," IEEE Sensors Journal, vol. 16, no. 20, pp. 7373-7380, 2016.

[14] http://www.matrikonopc.cn/opc-ua/

[15] M. Nicoleta, and K. Eniko, "Towards Lexicalization of DBpedia Ontology with Unsupervised Learning and Semantic Role Labeling,” IEEE 2016 18th International Symposium on Symbolic and Numeric Algorithms for Scientific Computing (SYNASC), pp. 256-263, 2016.

[16] F. Nurul, and I. Shofi, "Development of SPL Government System with Ontology Web Language,” IEEE International Conference on Cyber and IT Service Management, pp. 1-4, 2016.

[17] F. Zhang, F. Xue, and Y. Xu, "Collaborative modeling method of performance prototype for aerospace products based on ontology". Computer Integrated Manufacturing Systems, vol. 22, no. 8, pp. 1887-1899, 2016.

[18] G. Fang, Y. Guo, H. Liao, and L. Wang, "Knowledge representation and annotation method based on ontology for complex products design," Computer Integrated Manufacturing Systems, vol. 22, no. 9, pp. 2063-2071, 2016.

[19] Z. Shu, J. Wan, D. Li, J. Lin, A. Vasilakos and M. Imran, "Security in Software-Defined Networking: Threats and Countermeasures," Mobile Networks and Applications, vol. 21, no. 5, pp. 764-776, 2016. 